Paediatr Paedolog 2015 • [Suppl 2]: 50:S25-S28 DOI 10.1007/s00608-015-0304-1

Online publiziert: 2. September 2015

○) Springer-Verlag Wien 2015
Franz Waldhauser ${ }^{1} \cdot J^{\prime}$ hann Deutsch ${ }^{2} \cdot$ Sonia Gobara $^{3}$

1 Politische Kindermedizin, Wien, Österreich

${ }^{2}$ Politische Kindermedizin, Graz, Österreich

${ }^{3}$ Politische Kindermedizin, St. Pölten, Österreich

\title{
Kompetenzzentren und Versorgungsnetzwerke für Kinder und Jugendliche mit seltenen Erkrankungen
}

Das vorliegende Supplement enthält Analysen und Überlegungen zur Therapieoptimierung für Kinder und Jugendliche mit seltenen, komplexen und diagnostisch oder therapeutisch aufwendigen Erkrankungen. Weiters berichten Pädiater aus dem näheren Ausland (Niederlande, Schweiz, Deutschland), die dort im obersten Gesundheitsmanagement mitwirken, über die im Ausland bereits angewendeten organisatorischen Lösungsansätze und die dabei auftretenden Probleme.

Schließlich werden entsprechende Intentionen in Österreich vorgestellt - wie sie sich in dem jüngst veröffentlichten Nationalen Aktionsplan für seltene Erkrankungen (NAP.se) finden. Die Eingliederung dieser österreichischen Intentionen in die Bestrebungen der Europäischen Union (EU) und eine erste Stellungnahme eines prominenten Landespolitikers werden ebenfalls dargestellt. In Österreichs föderalem Gesundheitswesen ist die Durchführung derartiger Maßnahmen durch die Verfassung den Ländern zugeteilt. Schließlich wird die Therapieoptimierung für die österreichischen Kinder mit Krebserkrankungen als ein Beispiel dafür vorgestellt, wie einer derartigen Patientengruppe durch freiwillige Selbstorganisation der Behandler optimal geholfen werden kann. Österreichs Kinder mit diesen Erkrankungen haben die besten Überlebenschancen in Europa.

Für eine größere Zahl von Pädiatern, die oft jahrelang in die Tertiärversor- gung von österreichischen Kindern involviert waren bzw. sind, ist die Diagnoseund Versorgungssituation für Patienten mit seltenen Erkrankungen (SE) national [1] und international inakzeptabel. Daher haben sie sich in einer Arbeitsgruppe (AG) zusammengeschlossen, die mithelfen will, diese Situation im eigenen Land möglichst rasch einer Lösung zuzuführen. Die AG selbst hat letztlich im Verein Politische Kindermedizin ihre Heimat gefunden.

Gemeinsam mit anderen Interessenten - der European Paediatric Association, der Gesundheit Österreich $\mathrm{GmbH}$ (GÖG), dem Hauptverband der österreichischen Sozialversicherungsträger und der Dachorganisation der PatientenSelbsthilfegruppen für SE Österreichs (Pro Rare Austria) - haben sie im November 2014 in Salzburg ein Joint Meeting zum Thema „Kompetenzzentren und Versorgungsnetzwerke für Kinder und Jugendliche mit seltenen, diagnostisch und therapeutisch aufwendigen Erkrankungen“ organisiert.

Die hier vorliegenden Beiträge sind im Wesentlichen die Verschriftlichung der dort gehaltenen Referate. Es ist eine spezifische Leistung der Referenten, die oft entscheidende Management- und/ oder medizinische Leitungsfunktionen ausüben, dass sie neben ihrer ursprünglichen Tätigkeit zusätzlich Zeit für die Referate und besonders deren Verschriftlichung aufwenden konnten. Es zeigt, wie viel ihnen an diesem Thema liegt. Ihnen, aber auch allen beteiligten Insti- tutionen und den Kongressteilnehmern danken wir zu tiefst für ihren Input und ihr Engagement. Nicht zuletzt danken wir allen Sponsoren und den inserierenden Firmen, ohne deren Unterstützung der Kongress und die Publikation dieses Supplements in Pädiatrie \& Pädologie nicht möglich gewesen wären. Die Mitarbeiter des Springer-Verlags waren - wie in der Vergangenheit - verlässliche und geduldige Partner und Ratgeber. Dafür gehört ihnen unsere persönliche Dankbarkeit.

》) Der Nationale Aktionsplan für seltene Erkrankungen wurde im Februar 2015 implementiert

Während der Produktion dieses Bandes ist ein höchst relevantes Ereignis eingetreten, dem wir glücklicherweise noch voll Rechnung tragen konnten: Das Österreichische Bundesministerium für Gesundheit (BMG) hat den bei der GÖG in Auftrag gegebenen Nationalen Aktionsplan für seltene Erkrankungen (NAP.se) [2] am 28.2.2015 implementiert. Damit wird einerseits einer Vorgabe der EU nachgekommen und andererseits ein Rahmen geschaffen, der tatsächlich die Errichtung von Expertisezentren und deren Vernetzung ermöglichen sollte. Die Umsetzung müsste jetzt zügig in Angriff genommen werden. Dabei sollen Probleme, die in anderen Ländern beobachtet wurden, nach Möglichkeit vermieden werden. Deutschland und die Schweiz sind in der Umsetzung 
bereits etwas weiter vorangeschritten, weshalb die Erfahrungen dieser Länder von besonderem Interesse sind.

》) Seltene Erkrankungen haben bei vielen Pädiatern einen sehr hohen Stellenwert

Die große Herausforderung liegt in der enormen Vielzahl der SE mit mehr als 7000 verschiedenen Diagnosen und in der dadurch bedingten Unkenntnis der Details in weiten Kreisen der Ärzteschaft; gleichzeitig muss aber festgehalten werden, dass die SE bei vielen Pädiatern und damit befassten Kinderchirurgen bereits einen sehr hohen Stellenwert besitzen [3] Die im Vergleich zu anderen Ärztegruppen zahlenmäßig starke Präsenz der Pädiater auf der Österreichischen EuroplanKonferenz zur Umsetzung des NAP.se [4] im BMG am 22.5.2015 mag als Beleg dafür dienen. Trotzdem benötigt die Stellung einer Diagnose in diesem Bereich durchschnittlich noch Jahre [1], was für die betroffenen Familien einen sehr langen Leidensweg und eine lange Zeit bis zu einer dem Stand der Wissenschaft entsprechenden Versorgung bedeutet. Auf der anderen Seite macht gerade die Vielzahl der verschiedenen Erkrankungen den Bereich der SE zu einem Modellfall für die personalisierte Medizin (A. Grüters-Kieslich). Wie in Deutschland ist auch in Österreich der Ruf der Stunde daher der Ruf nach einem Zusammenschluss der beteiligten Kollegen und Zentren, um letztlich die Politiker von den aus dem NAP.se resultierenden Notwendigkeiten zu überzeugen.

Abschließend möchten wir R. Riedl, den Obmann von Pro Rare Austria, zitieren: „Für die Umsetzung der Maßnahmen braucht es mutige Gesundheitspolitiker/-innen und den guten Willen aller Partner im Gesundheitssystem “ [5]. Sein deutsches Pendant A. Reimann von der Allianz Chronischer Seltener Erkrankungen (ACHSE) meinte jüngst, dass Papier geduldig ist, entscheidend aber die Umsetzung der geplanten Maßnahmen sei ( „... von der Zufälligkeit zur Verlässlichkeit..." [3]). Dass dabei in einem föderalen System den Ländern eine besondere Verantwortung zukommt, steht außer
Zweifel. Dass sie in einem föderalen System auch wahrgenommen werden kann, zeigt die Entwicklung in der Schweiz.

Die Herausgeber

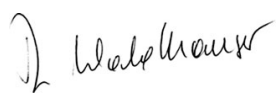

F. Waldhauser

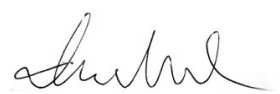

J. Deutsch

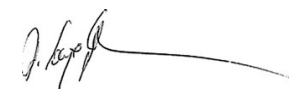

S. Gobara

Wien, im August 2015

\section{Korrespondenzadresse}

a. o. Univ.-Prof. Dr. F. Waldhauser

Politische Kindermedizin

Amerlingstrasse 4/20, 1060 Wien, Österreich

f.waldhauser@aon.at

Interessenkonflikt. F. Waldhauser, J. Deutsch und

S. Gobara geben an, dass kein Interessenkonflikt besteht.

\section{Literatur}

1. Voigtländer $T$, Bachner $F$, Unterberger $U$, Leopold C, Ladurner J, Habl C (2012) Seltene Erkrankungen in Österreich. Empirische Erhebung zur aktuellen Situation von Betroffenen; Ergebnisbericht. NKSE der Gesundheit Österreich GmbH/BMG, Wien. http://www.goeg.at/cxdata/media/download/ berichte/seltene_erkrankungen.pdf

2. BMG (2015) Nationaler Aktionsplan für Seltene Erkrankungen NAP.se 7 2014-2018. Erstellt von der Nationalen Koordinationsstelle für Seltene Erkrankungen (NKSE) im Auftrag des BMG. BMG, Wien. http://www.bmg.gv.at/cms/home/ attachments/7/6/1/CH1075/CMS1424876546854/ nap_selteneerkrankungen_2015.pdf

3. DGKJ-Kolloquium in Zusammenarbeit mit der AG ZSE (2015) Entwicklung der Pädiatrischen Bereiche der Zentren für Seltene Erkrankungen (ZSE). Berlin 25.6.2015

4. Gesundheit Österreich GmbH (2015) Nationale EUROPLAN-Konferenz. http://www.goeg.at/de/ Bereich/ab/396.html

5. Riedl R (2015) Die Erwartungen von Patient/innen mit seltenen Erkrankungen an die Versorgungsstruktur. Pädiatrie Pädologie 50. doi: 10.1007/s00608-015-0275-2 


\section{Hier steht eine Anzeige.}

第 Springer 


\section{Hier steht eine Anzeige.}

第 Springer 